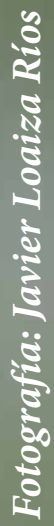

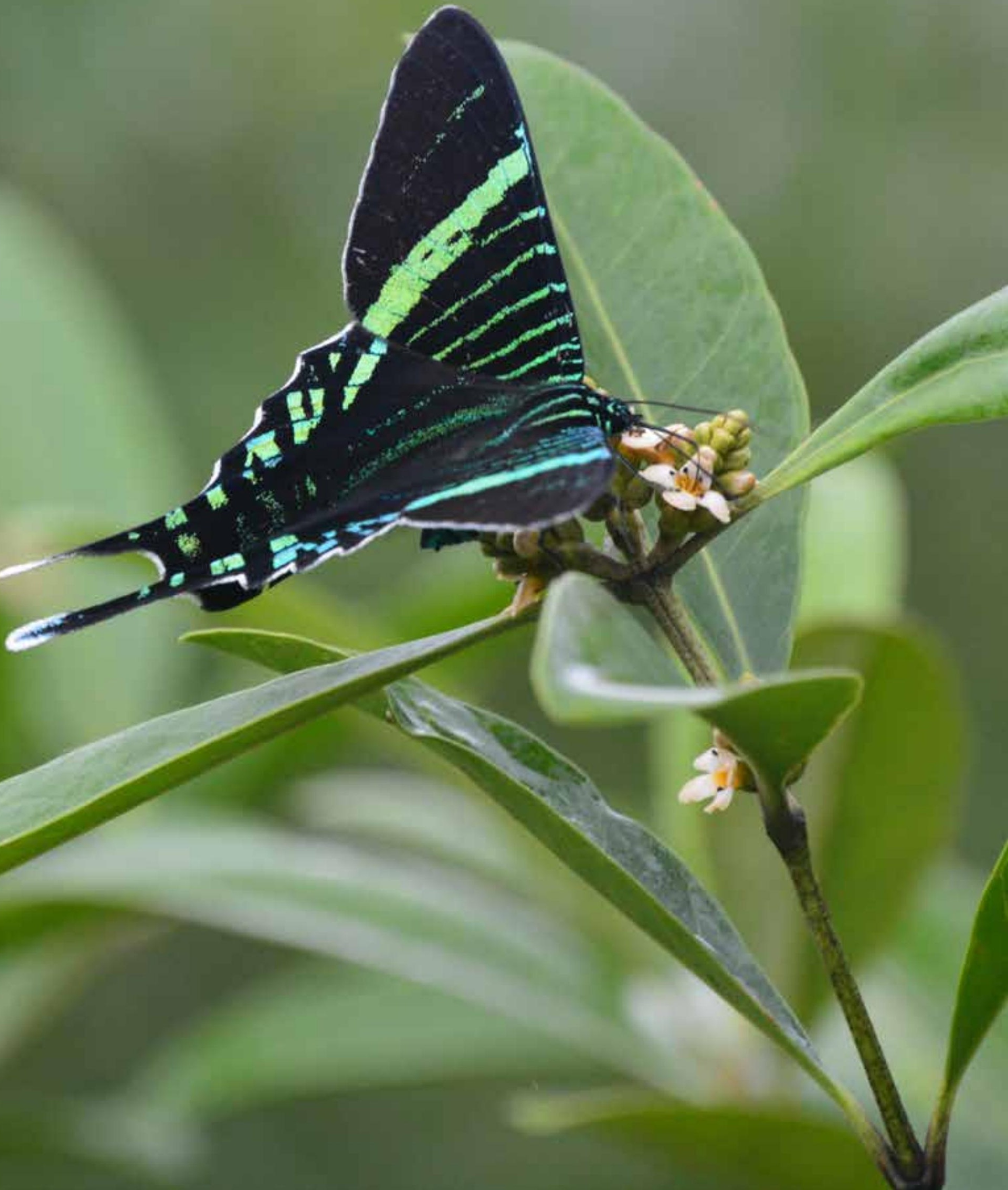




\title{
Caracterización de ideas previas sobre el concepto de ecosistema en estudiantes del grupo 505 del Colegio Minuto de Dios, ubicado en Ciudad Verde, Soacha- Cundinamarca \\ Characterization of Preliminary Ideas on Ecosystem Concept in Grade 505 Students from Minuto de Dios School, Located in Ciudad Verde, Soacha, Cundinamarca
}

\author{
Kelly Alejandra Motta Montiel ${ }^{1}$ \\ Andrea Del Pilar Uyaban Sandoval ${ }^{2}$
}

Fecha de recepción: 5 de abril de 2016

Fecha de aprobación: 15 de octubre de 2016

\section{Resumen}

El presente trabajo se fundamentó en la unidad didáctica Eco-vida, comprendiendo los ecosistemas, desarrollada durante tres sesiones de una hora y 50 minutos en el grupo 505 del Colegio Minuto de Dios (Ciudad Verde, Soacha, Cundinamarca). Tiene como propósito categorizar las ideas previas que los alumnos tienen respecto al concepto de ecosistema como proceso de investigación bajo el paradigma hermenéutico y la metodología de carácter cualitativo. Se reflexiona a partir de los dibujos y los escritos plasmados por los estudiantes acerca de los ecosistemas, apoyando los criterios en tres niveles: la propuesta del nivel 0 , donde los estudiantes dan respuestas con objetos que no se relacionan con la interacción de los componentes de un ecosistema; el nivel 1, donde los estudiantes proporcionan ideas alejadas de la configuración de un ecosistema, y el nivel 2, donde los estudiantes logran dar una introducción a temas como biocenosis, biotopo y red trófica.

Palabras clave: ideas previas, ecosistema, categorización

\section{Abstract}

This paper is based on the teaching unit Ecovida, understanding ecosystems developed during three sessions of one hour and 50 minutes in the group 505 of Minuto de Dios School (Green City, Soacha-Cundinamarca). It aims to categorize the preconceptions that students have about ecosystem concept as research process under the hermeneutical paradigm and qualitative methodology. Authors reflect based on students' drawings and writings about ecosystems, supporting the criteria on three levels: level 0 proposal, where students give answers with items that are not related to the ecosystem components interaction; level 1, where students provide remote ideas about configuration of an ecosystem, and level 2, where students are able to give an introduction to topics such as biocenosis, biotope and food web

Keywords: pre ideas, ecosystem, categorization

1 Estudiante, Licenciatura en Biología, Universidad Distrital Francisco José de Caldas, (Colombia). Correo electrónico: Kellyaleja_629@ hotmail.com

2 Estudiante, Licenciatura en Biología, Universidad Distrital Francisco José de Caldas (Colombia). Correo electrónico: aduyabans@correo. udistrital.edu.co 


\section{Introducción}

La unidad didáctica Eco-vida comprendiendo los ecosistemas se desarrolló en el grado quinto, grupo 505 , de la jornada de la tarde del Colegio Minuto de Dios (ubicado en Ciudad Verde, Soacha, Cundinamarca). Para lograr una contextualización en la planeación de actividades de acuerdo con la realidad escolar allí evidenciada, se caracterizó a la docente de ciencias naturales y a los estudiantes (cuyas edades oscilan entre los 10 y los 12 años) de manera conjunta con la caracterización institucional, lo que nos permitió empalmar el modelo pedagógico con los objetivos de nuestra unidad, lo que, como plantean Perkins y Blythe (2005), nos invita a superar las barreras del memorismo, del actuar rutinario y del pensamiento bancario. Para ello, se implementó la primera parte de la unidad didáctica durante tres sesiones - cada una, de una hora y 50 minutos-, y surgió la pregunta que guía nuestra investigación: ¿Cuáles son las ideas previas que tienen los estudiantes del grupo 505 del Colegio Minuto de Dios sobre el concepto de ecosistema?

Con una perspectiva constructivista, se estima que el conocimiento es construido por el individuo que interactúa con su entorno, por lo cual son importantes y se deben tener en cuenta aspectos como los psicológicos, los culturales y los sociales. El sujeto entiende el mundo a través de sus propias experiencias, ideas personales que le sirven de base para interpretar e integrar las nuevas informaciones. Así pues, el aprendizaje no es tan simple como un proceso de adicionar información, sino, al contrario, es el proceso de reestructurar la información nueva con las ideas previas que el estudiante pueda tener. De ahí la importancia de determinar las ideas que tienen los alumnos acerca de los fenómenos naturales, porque dichas ideas, o esquemas previos, le sirven para interpretar lo que se le enseña (Grilo, Mellado y Ruiz, 2004).

En la variedad de terminología usada al hablar de la relación entre el conocimiento escolar de los alumnos y el conocimiento cotidiano, centramos nuestra atención en las ideas previas, más que en concepciones falsas, preconceptos y representaciones alternativas; así, nos permitimos citar a Cubero (1997), quien plantea:

El hecho de reconocer que los niños tienen ideas, que saben cosas aunque no se las hayamos enseñado en la escuela, es como caer en cuenta de que efectivamente esos conocimientos están allí, es como designar algo que hasta ese momento no se había considerado. (p. 1721).
Por otro lado, Bello (2004) plantea que las ideas previas:

Son construcciones que los sujetos elaboran para dar respuesta a su necesidad de interpretar fenómenos naturales o conceptos científicos, y para brindar explicaciones, descripciones o predicciones. Son construcciones personales, pero a la vez son universales y muy resistentes al cambio; muchas veces persisten a pesar de largos años de instrucción escolarizada. (p. 210).

Teniendo en cuenta que, como lo menciona Rincón (2011), la enseñanza "del concepto ecosistema y conceptos relacionados con su dinámica como flujos de energía, ciclos de nutrientes, redes tróficas, interacciones, estabilidad, equilibrio y efecto de las perturbaciones sobre la dinámica del ecosistema son fundamentales" (p. 78), tomamos este concepto como central para la comprensión del funcionamiento de la naturaleza, que ayuda a establecer una visión compleja, dinámica y relacionada de ella, que permite superar algunos de los problemas centrales en el tratamiento tradicional de los contenidos de la biología y, a la vez,

permite el conocimiento de los seres vivos que conforman un determinado ecosistema, hace entrever las relaciones de interdependencia que se establecen entre ellos, y a su vez, la importancia de mantener cada uno de los eslabones de la cadena alimenticia, como las condiciones físicas del entorno para conservar el equilibrio ecológico. (Casillas y Ramos, 2012, p. 14).

Así pues, consideramos de suma importancia que los alumnos puedan comprender el concepto ecosistema, pues sin dicho referente resultará muy difícil que puedan valorar los cambios introducidos por el hombre; esto, sin dejar de lado las ideas previas que serán el punto de partida y de referencia hacia la introducción de nuevos conceptos. Es muy importante conocer las ideas de los alumnos y reflexionar en torno a estas, a la vez que, como lo menciona Pozo (1996), "el tratamiento curricular de las ideas previas de los alumnos no es tanto dirigido a que sean abandonadas y sustituidas por conocimientos científicos, sino que se integren en estructuras de conocimientos más complejas y próximas a las teorías científicas" (p. 1)

\section{Marco teórico}

"Se denominan ideas previas a las concepciones que tienen los estudiantes sobre diferentes fenómenos, aún sin recibir ninguna enseñanza sistemática al respecto; estas ideas se crean a partir de las experiencias cotidianas" (Rayas, 2002, p. 1). 
Para denominar las ideas previas existen muchos conceptos; algunos de ellos son: ideas intuitivas, ciencia de los niños, representaciones de los alumnos (Osborne y Gilbert, 1983). Errores conceptuales por Helm (2000), Preconcepciones por Novak, (1983); representaciones por Giordan (1982), preconceptos, Mc Dremott (1984), y Duit (1984) citados por Rayas, (2002). Todas estas concebidas como elementos determinantes en el aprendizaje y la enseñanza en las ciencias.

Las ideas previas responden a una lógica de pensamiento, influenciada por las experiencias de la vida cotidiana; generalmente, son distintas de los conocimientos científicos y escolares. Los sujetos van conformando explicaciones sobre la realidad de manera coherente, lo cual hace que las ideas previas puedan persistir aún después de la enseñanza (Rayas, 2002).

Las características del pensamiento influyen de manera determinante en las ideas previas, de manera que estas parecen evolucionar a medida que los niños se van adaptando a experiencias más amplias y se van desarrollando hacia un pensamiento más formal; las ideas previas se van modificando, aunque en casos como el de los conceptos de Ciencias Naturales, que presentan cierta complejidad, las ideas previas prevalecen aún en la edad adulta (Rayas, 2002).

El enfoque de ideas previas dentro de la educación
tiene sus antecedentes a partir de la teoría de Ausubel
(1963) donde refiere el concepto de "aprendizaje sig-
nificativo" señalando la importancia que tienen los
conocimientos previos. Anteriormenteya se había con-
templado la incidencia de los conocimientos previos
por Bartlett (1932) y Kelly (1955), pero esta tendencia
adquiere mayor auge con las investigaciones realizadas
por Ausubel durante los años setentas, en las cuales
aparecen los conocimientos previos como fundamento
de la significatividad en el aprendizaje; otros autores
realizan estudios retomando este planteamiento,
destacando que los alumnos, antes de acceder a la
instrucción formal, han desarrollado ideas que preva-
lecen aún con la enseñanza formal. (Rayas, 2002, p. 21).

La valoración de las ideas previas ha permitido, en primer lugar, que se tenga conocimiento acerca de las concepciones con las cuales los estudiantes enfrentan el aprendizaje de los conocimientos científicos. En segundo lugar, pone de manifiesto que dicho aprendizaje lleva incorporado un interesante problema de transformación conceptual, y, por lo tanto, tal proceso no sería una adquisición exnihilo. En tercer y último lugar, la existencia de las ideas previas hace evidente el desafío de enfrentar los procesos de aprendizaje -y, en especial, la didáctica- bajo una óptica distinta, pues para producir el cambio conceptual no bastaría solo con tomar en consideración dichas ideas previas, si, acaso, este acto no va acompañado de una metodología de enseñanza que incorpore la incertidumbre, la presencia de dudas y conjeturas, la consideración de soluciones alternativas, el descarte de respuestas muy rápidas y seguras, basadas en un mero sentido común, así como por tratamientos específicos con falta de coherencia en el análisis de diferentes situaciones (Gil, 1993).

Algunas de las principales características de las ideas previas han sido detalladas por Pozo, Asencio y Carretero (1989), así:

- Ser espontáneas: surgen en forma natural y a partir de la interacción de quien aprende con el mundo y con la gente.

- Tratarse de construcciones personales; es decir, producto de la experiencia personal con el mundo, y no a través de una escolaridad formal.

- Desde el punto de vista formal de la ciencia, se trata de ideas incorrectas; sin embargo, son verosímiles en un contexto cotidiano extraescolar.

- Encontrarse implícitas en quien aprende; por ello, no es fácil exteriorizarlas ni verbalizarlas.

- Presentarse de forma incoherente o ser contradictorias entre sí.

- Ser resistentes al cambio, debido a que tienen el carácter de verdades indiscutibles, pues se basan en la epistemología del sentido común (Gil y Guzmán, 1993).

- Ser compartidas por personas de muy diversas características (edad, país, formación), pese a ser construcciones personales, cuestión que llega a trascender en el tiempo.

- Ser dominadas por la percepción; en general, lo que se ve es lo que se cree.

Si bien son muchas las fuentes que explican la existencia de las ideas previas, los especialistas identifican dos grandes fuentes de origen. En primer lugar, se sitúan las generadas de forma directa en el sistema escolar formal, y, por lo tanto, relacionadas con la experiencia escolar de cada uno de los sujetos que aprenden; en este caso, se identifican como fuentes los libros de texto u otros materiales o recursos empleados en los estudios y la información entregada por los docentes, así como la interferencia del vocabulario científico, técnico y propio de cada disciplina en el lenguaje cotidiano (no son pocas las palabras con un significado distinto, según se trate de un escenario científico o de uno coloquial). En segundo lugar se sitúan aquellas cuyo origen se encuentra en el mundo no escolar o contextual; en este caso, se identifican como fuentes las experiencias y las observaciones de los propios sujetos 
respecto a la vida cotidiana, así como la cultura propia de cada grupo humano (creencias, usos, costumbres y prácticas cotidianas), y la influencia de los medios de comunicación (Muñoz, 2005).

Según lo planteado, basamos nuestro trabajo en las ideas previas que los estudiantes tienen acerca del concepto ecosistema; término, a su vez, introducido por Tansley (193) como un concepto ecológico holístico e integrativo que combina los organismos vivos y el ambiente físico en un sistema. "Los ecosistemas son de varios tipos y tamaños, representan una categoría en un sistema físico jerárquico desde el propio universo al átomo". (p. 345) Tansley presentó este concepto como una refutación de la ontología clementsiana, al afirmar:

Personalmente he dado mis razones para rechazar los términos "organismo complejo" y comunidad biótica. El término bioma primeramente propuesto por Clements para el complejo íntegro de organismos que habitan una cierta región, es inobjetable y conveniente para ciertos propósitos; si bien los organismos constituyen nuestro interés principal, no podemos separarlos de su ambiente especial, ya que con él conforman un sistema físico, representan las unidades básicas de la naturaleza, en cada sistema existe intercambio constante del más variado tipo, no solo entre los organismos, sino entre la parte orgánica e inorgánica. Estos ecosistemas, como nosotros podemos denominarlos, son de las más diversas clases y tamaños. (1935, p. 299).

Así pues, el concepto de ecosistema de Tansley (1935) identificó un sistema que era:

1. Un elemento en una jerarquía de sistemas físicos desde el universo hasta el átomo

2. El sistema básico de la ecología

3. Compuesto tanto de organismos como del ambiente físico

Según Tansley, un ecosistema está conformado por la biocenosis y su espacio; partiendo de estos conceptos como parte integrante, entendemos los biotipos como las formas típicas de los organismos.

Para López (2002), el biotopo es "el ambiente de vida y se aplica al espacio físico, natural y limitado, en el cual vive una biocenosis" (p. 139). Y siguiendo la definición de Mobius (1877) (como se cita en López, 2002), "una biocenosis es una comunidad de productores primarios y de heterótrofos que se regulan mutuamente y dependen del ambiente físico exterior a la biocenosis"; también es conocida como comunidad biótica o comunidad ecológica. Por su parte, para Ibarra y Gil (2009), la biocenosis es una comunidad o un conjunto de poblaciones de distintas especies, las cuales habitan un lugar geográfico determinado y están influenciados por factores físicos como la luz, la humedad, la temperatura, etc.

Respecto a los niveles de caracterización de las ideas previas, nos permitimos citar a García (2003) (citado por Rincón, 2011 p. 88), quien expone los niveles de progresión para el ecosistema:

Nivel 1: Donde prima una concepción de ecosistema como Medio Aditivo: Los estudiantes no reconocen una organización subyacente a lo perceptible, en donde las relaciones tróficas a lo sumo, si se explicitan, son binarias en un medio que es concebido como estático y en donde la diversidad de elementos presentes se queda en los más evidentes, donde las relaciones son sencillas y no configuran un ecosistema.

Desde esta concepción el medio es bien un medio-escenario, es decir es percibido por los alumnos como un fondo homogéneo e indiferenciado donde todo se entremezcla sin una organización aparente, bien un medio aditivo (Astolfi y Drouin, 1986) en el que éste se entiende como la mera suma de sus componentes. Esta concepción se manifiesta en los alumnos cuando aluden a un inventario de lo que hay en él, centrándose únicamente en la enumeración de sus componentes y cuando solo se refieren a algunas relaciones sencillas que se establecen entre éstos (García, 1999). Esta aditividad dificulta en gran medida la construcción de la noción de red trófica, pues solo se asume un camino posible, de manera que cada elemento se relaciona únicamente con el que le precede o el que va detrás en la cadena. (García, 2003, p.87).

Nivel 2: Ecosistema como Organización Simple: El ecosistema es considerado como un sistema de organización simple donde las cadenas tróficas son rígidas en un equilibrio estático. La diversidad de elementos presentes aún continúa siendo más evidentes en un medio donde las relaciones ecológicas son causales y sencillas. En este nivel se asume que hay una relación basada en relaciones causales, aunque sean propias de una causalidad mecánica y lineal. También se identifica una mayor diversidad de relaciones ecológicas: relaciones entre la biocenosis y el biotopo, relaciones interespecíficas, así como relaciones intraespecíficas. Además, se reconocen los descomponedores y algunos elementos abióticos. (p. 88).

Nivel 3: Ecosistema como Organización Compleja: El ecosistema es visto como un sistema de organización compleja donde se conciben niveles tróficos dentro 
de una red trófica dinámica y flexible y se maneja un equilibrio dinámico donde se presenta sucesiones ecológicas, ciclos biogeoquímicos y el concepto de nicho. Las relaciones ecológicas son entendidas como interacciones. En este nivel se reconoce que en el cambio de los ecosistemas se mantiene la organización básica de los mismos. (p. 88).

Se propone por parte de las autoras de este artículo el nivel 0 : No relación, como el nivel donde no se logra dar explicación acerca de los ecosistemas y se dan respuestas con objetos que no se relacionan con la interacción de los componentes de un ecosistema. El estudiante aborda, principalmente, objetos de su diario vivir, como lápices, bolsos y escritorios, y los introduce en un ecosistema, sin mencionar los factores abióticos como vitales para la subsistencia de un ser biótico, ni la relación entre estos, el entorno, la red trófica y los ciclos biogeoquímicos.

\section{Metodología}

Con el fin de fortalecer el conocimiento de los futuros docentes en formación, se propusieron el diseño y la implementación de una unidad didáctica, de la que posteriormente surge nuestro problema de investigación, enmarcado dentro del paradigma cualitativo; al respecto, Ricoy (2006) señala:

El carácter cualitativo que caracteriza al paradigma interpretativo busca profundizar en la investigación, planteando diseños abiertos y emergentes desde la globalidad y contextualización. Las técnicas de recogida de datos más usuales son la observación participativa, historias de vida, entrevistas, los diarios, cuadernos de campo, los perfiles, el estudio de caso, etc. Tanto las conclusiones como la discusión que generan las investigaciones que comparten la doctrina del paradigma interpretativo están ligadas fundamentalmente a un escenario educativo concreto contribuyendo también a comprender, conocer y actuar frente a otras situaciones. (p. 17).
La interpretación se hizo tomando en cuenta instrumentos tales como el diario de campo y el análisis de imágenes de las ideas previas que relatan las vivencias en el aula de clase durante la implementación de la unidad; estos instrumentos se utilizaron para el análisis de los resultados.

La implementación se llevó a cabo en el Colegio Minuto de Dios, ubicado en Soacha, con estudiantes del grado 505 de la jornada de la tarde. Entre estos se aplicó un cuestionario que se resolvió durante una sesión de clase de dos horas; cabe aclarar que la actividad estaba diseñada para conocer las ideas previas que los alumnos tenían respecto al concepto de ecosistema. Tomando como población el total de los alumnos de dicho grado, participaron 39 estudiantes.

Para la caracterización de las ideas previas nos enmarcamos dentro de los niveles planteados por García (2003), quien propone en tal sentido:

Nivel 1: donde prima una concepción de ecosistema como medio aditivo.

Nivel 2: ecosistema como organización simple.

Nivel 3: ecosistema como organización compleja.

Además de los anteriores niveles, nos permitimos agregar el nivel 0: no relación.

\section{Resultados}

\section{¿Qué es un ecosistema?}

En total, se identificaron 15 puntos de vista de los niños respecto a la pregunta, de ellos, el $29 \%$ estuvieron de acuerdo en relacionar el ecosistema con el lugar donde viven los animales y las plantas. Cabe resaltar que en cuanto a esta pregunta, el segundo porcentaje más representativo estuvo en manos de los niños que no respondieron la pregunta, con el 13\%. El 7\% de los alumnos asociaron la palabra en estudio a los seres vivos, a todo lo que nos rodea y al cuerpo humano y sus órganos.

Tabla 1. Relación de los niveles y las respuestas de los estudiantes a la pregunta: ¿Qué es un ecosistema?

\begin{tabular}{|c|c|c|c|c|}
\hline Nivel & $\begin{array}{l}\text { Número de } \\
\text { estudiantes }\end{array}$ & Porcentaje & Justificación & $\begin{array}{c}\text { Respuestas de los } \\
\text { estudiantes }\end{array}$ \\
\hline 0 (no relación) & 13 & $33,33 \%$ & $\begin{array}{l}\text { El niño no logra dar explicación acerca } \\
\text { de los ecosistemas, y da respuestas con } \\
\text { palabras que no se relacionan con la inte- } \\
\text { racción de los componentes de un ecosis- } \\
\text { tema. }\end{array}$ & $\begin{array}{l}\text {-Algo relacionado con otras cosas } \\
\text {-El cuerpo humano con sus órganos } \\
\text {-Fábrica. } \\
\text {-Universo o galaxia } \\
\text {-Algo "de Sociales" } \\
\text { Pregunta no respondida. }\end{array}$ \\
\hline
\end{tabular}




\begin{tabular}{|c|c|c|c|c|}
\hline Nivel & $\begin{array}{l}\text { Número de } \\
\text { estudiantes }\end{array}$ & Porcentaje & Justificación & $\begin{array}{l}\text { Respuestas de los } \\
\text { estudiantes }\end{array}$ \\
\hline $\begin{array}{l}1 \text { (ecosistema como } \\
\text { medio aditivo) }\end{array}$ & 19 & $48,71 \%$ & $\begin{array}{l}\text { El estudiante no logra configurar un eco- } \\
\text { sistema, y lo define a partir de la suma de } \\
\text { componentes. }\end{array}$ & $\begin{array}{l}\text {-Seres vivos } \\
\text {-Bosque } \\
\text { Cuidar la naturaleza } \\
\text {-Donde viven los animales y las plantas }\end{array}$ \\
\hline $\begin{array}{l}2 \text { (ecosistema como } \\
\text { organización simple) }\end{array}$ & 7 & $17,94 \%$ & $\begin{array}{l}\text { La diversidad de elementos presentes } \\
\text { sigue siendo la más evidente, pero se logra } \\
\text { relacionar a continuación los descompo- } \\
\text { nedores, los seres abióticos y algún tipo de } \\
\text { relación inter o intraespecífica. }\end{array}$ & $\begin{array}{l}\text {-Relación entre seres vivos y muertos } \\
\text { - Grupo de animales } \\
\text { - Cuando un animal se come a otro } \\
\text {-Seres vivos y no vivos }\end{array}$ \\
\hline
\end{tabular}

Fuente: elaboración propia.

\section{¿Qué tiene un ecosistema?}

Para la respuesta a esta pregunta, en la que se les pidió a los estudiantes que dibujaran los componentes de un ecosistema, se obtuvo como resultado que el $100 \%$ de los niños hicieron dibujos en cuyo principal componente se encuentran cosas vivas y cosas no vivas; a partir de ello, en todos los casos se encuentran dibujos de cosas vivas, dentro de las cuales, el $58 \%$ de los participantes dibujaron plantas; el 68\%, animales, y el 58\%, personas (cabe aclarar que los porcentajes suman más del $100 \%$, debido a que algunos niños dibujaron más de un objeto vivo).
En cuanto a los dibujos de objetos no vivos, el $26 \%$ de los niños dibujaron juguetes e instrumentos musicales; el $28 \%$, muebles (sillas y mesas) y rocas; el $18 \%$, útiles escolares, y el 2\%, ropa. El 5,12\% de los estudiantes asociaron las cosas no vivas a cosas que estaban vivas, pero murieron.

Dentro de los dibujos hechos por los estudiantes, los que más se repitieron fueron: de los animales, el gato, con el $21 \%$; de las plantas, el árbol, con el 59\%, y de los útiles escolares, el cuaderno, con el 39\%.

Tabla 2. Niveles y respuestas de los estudiantes a la pregunta: ¿Qué tiene un ecosistema?, en relación con las cosas "vivas".

Dibujos de cosas "vivas"

\begin{tabular}{|l|c|c|l|l|}
\hline \multicolumn{1}{|c|}{ Nivel } & $\begin{array}{c}\text { Número de } \\
\text { estudiantes }\end{array}$ & Porcentaje & Justificación & $\begin{array}{c}\text { Respuestas de los } \\
\text { estudiantes }\end{array}$ \\
\hline $\begin{array}{l}1 \text { (ecosistema como } \\
\text { medio aditivo) }\end{array}$ & 39 & $100 \%$ & $\begin{array}{l}\text { El estudiante plasma en sus dibujos los animales y } \\
\text { las plantas como factores principales en el ecosis- } \\
\text { tema; sin embargo, se observa una intervención } \\
\text { antrópica en 22 de los estudiantes. }\end{array}$ & $\begin{array}{l}\text {-Pnimales } \\
\text {-Personas }\end{array}$ \\
\hline
\end{tabular}

Tabla 3. Niveles y respuestas de los estudiantes a la pregunta: ¿Qué tiene un ecosistema?, en relación con las cosas "no vivas".

\section{Dibujos de cosas "no vivas"}

\begin{tabular}{|c|c|c|c|c|}
\hline Nivel & $\begin{array}{l}\text { Número de } \\
\text { estudiantes }\end{array}$ & Porcentaje & Justificación & $\begin{array}{c}\text { Respuestas de los } \\
\text { estudiantes }\end{array}$ \\
\hline 0 (no relación) & 26 & $66,66 \%$ & $\begin{array}{l}\text { El estudiante no logra configurar la idea de un eco- } \\
\text { sistema con respecto a un biotopo, y plasma en sus } \\
\text { dibujos objetos de su cotidianidad que considera } \\
\text { objetos no vivos, pero enmarcados con una posible } \\
\text { función en el ecosistema. }\end{array}$ & $\begin{array}{l}\text {-Juguetes } \\
\text {-Muebles } \\
\text {-Ropa } \\
\text {-Útiles escolares } \\
\text {-No dibujo }\end{array}$ \\
\hline $\begin{array}{l}1 \text { (ecosistema como } \\
\text { medio aditivo) }\end{array}$ & 13 & $33,33 \%$ & $\begin{array}{l}\text { El estudiante logra relacionar los factores abióticos, } \\
\text { pero como la mera suma de componentes, donde } \\
\text { no logra dar cuenta de qué función cumplen las } \\
\text { rocas o los esqueletos en un ecosistema. }\end{array}$ & $\begin{array}{l}\text {-Esqueleto } \\
\text {-Rocas }\end{array}$ \\
\hline
\end{tabular}

Fuente: elaboración propia. 
Tabla 4. Relación de los niveles y las respuestas de los estudiantes a la solicitud: Dibuja un ecosistema.

Dibuja un ecosistema

\begin{tabular}{|c|c|c|c|c|}
\hline Nivel & $\begin{array}{l}\text { Número de } \\
\text { estudiantes }\end{array}$ & Porcentaje & Justificación & Respuestas \\
\hline $\begin{array}{l}1 \text { (ecosistema como } \\
\text { medio aditivo) }\end{array}$ & 27 & $69,23 \%$ & $\begin{array}{l}\text { Los alumnos que se ubican en esta } \\
\text { categoría se caracterizan por darles } \\
\text { más importancia a los animales que a } \\
\text { las plantas, y asíse mencionan elemen- } \\
\text { tos del biotopo. Consideran al "ecosis- } \\
\text { tema" una organización aditiva, en la } \\
\text { cual son más relevantes los elementos } \\
\text { que las relaciones (García, 2003). }\end{array}$ & 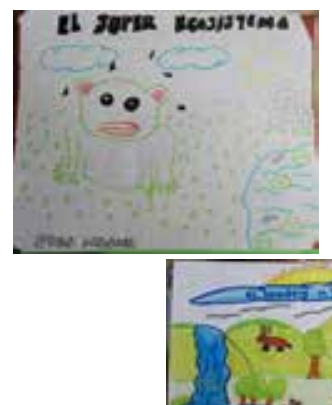 \\
\hline $\begin{array}{l}2 \text { (ecosistema } \\
\text { como organización } \\
\text { simple) }\end{array}$ & 12 & $30,76 \%$ & $\begin{array}{l}\text { En este nivel se encuentran los estu- } \\
\text { diantes que reconocen explíitamente } \\
\text { las relaciones que dan lugar a configu- } \\
\text { raciones simples del tipo de las cade- } \\
\text { nas tróficas (García, 2003). }\end{array}$ & 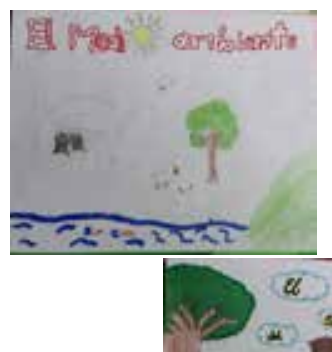 \\
\hline
\end{tabular}

Fuente: elaboración propia.

\section{Análisis de resultados}

Respecto a los dibujos realizados por los niños sobre las cosas que están vivas y las que no, podemos inferir que sus dibujos de cosas no vivas implican cosas de su diario vivir, como sillas y útiles escolares, pero no dan cuenta de la interacción del biotopo y la biocenosis en un ecosistema. Por su parte, en los niños que logran dar cuenta de que un ecosistema está compuesto por plantas y animales, encontramos que el $58 \%$ de los niños dibujó plantas, y el 68\%, animales (se destacan el perro, el gato y el pez), lo que da cuenta de que "La prevalencia dada al reino animal y para el caso de las representaciones gráficas (animales domésticos) se debe principalmente al carácter utilitario que los estudiantes le dan a los mismos" (Rincón, Medellín y Vargas, 2004, citados por Rincón, 2011, p. 78). En cuanto a los dos estudiantes que asociaron las cosas no vivas a cosas que estaban vivas, pero murieron, podemos rescatar el hecho de que, posiblemente, ya tengan un conocimiento acerca de los organismos descomponedores y de la red trófica, pero, a la vez, puede que ellos crean que dichos organismos que ya no están vivos ya no cumplen ninguna función en el ecosistema.

Podemos inferir que ninguno de los estudiantes tuvo una visión micro de los componentes de un ecosistema; en cuanto a los niños que dibujaron plantas, cabe preguntarnos si podrían estar relacionando respiración y fotosíntesis, o si "Los niños no reconocen que la fotosíntesis es el proceso mediante el cual la energía del ambiente llega a estar disponible para las plantas, y, posteriormente, para los animales" (Leach, Driver, Scott y Wood-Robinson, 1992, citados por Rincón, 2011 p.78)

Fijamos nuestro interés en la valoración de los planteamientos frecuentes con la perspectiva antropológica, al hablar de la inclusión del hombre en los dibujos (esencial en muchos de los gráficos), donde se reconoce que es importante aclarar si estos piensan que el hombre es parte de los ecosistemas, si cumple alguna función en un ecosistema y si se encuentran divergencias con el estudio realizado por Leach et al. (1992), citados por Rincón, 
(2011), y quien nos da una valoración frecuente con la perspectiva teleológica y antropológica en niños entre los 5 y los 7 años, pero poco común en niños mayores de 9 años.

Respuestas en las que el $40 \%$ de los niños expone que "los seres vivos se sirven de otros para realizar sus actividades" fueron ubicadas en el nivel 2 , considerando que nos permiten introducir conceptos como red trófica; lo contrario pasaría con los niños para quienes "los seres vivos no se relacionan en nada" (ubicados en el nivel 1), pues podrían, además, caer en el error de abordar los componentes de una biocenosis con una perspectiva de individuo y $\sin$ ninguna relación con su biotopo.

Se encontró que los estudiantes relacionan el ecosistema con la naturaleza y el medio que los rodea, pues en este incluyen animales y plantas. Además, mencionan que hay una relación entre estos, es decir, para ellos hay una interacción, algo muy parecido a lo que señalan Sánchez y Pontes (2009); ello indica que, para la mayor parte de los alumnos, un ecosistema es un lugar donde viven juntos muchos seres vivos. Por otra parte, encontramos respuestas tales como las que hallan relación entre seres vivos y muertos, y cuando un animal se come a otro. Tal tipo de respuestas nos puede dar un breve acercamiento a lo que sería el nicho ecológico, así como sus relaciones con otras especies y por su forma de alimentación.

Tomando en cuenta lo que nos postula García (2003) para la caracterización de las respuestas de los alumnos, podemos inferir que los estudiantes se ubicaron en el nivel 1 , ya que en sus dibujos reconocen los seres vivos como un componente importante, pero no reconocen su papel organizador en un ecosistema. De hecho, los alumnos que se sitúan en este modelo solo mencionan seres próximos a su propia experiencia, e ignoran que hay componentes de tanta relevancia como los seres microscópicos. Además, conceden más valor a los animales que a las plantas, y mencionan elementos del biotopo, se alude a los aspectos más visibles y tangibles de este - tierra, agua, rocas- (García, 2003), datos muy parecidos a los de nuestra investigación, ya que los niños sujetos de estudio en ella dieron respuestas con objetos que eran muy cercanos a su propio contexto, como es el caso de los animales (mencionaron gatos y perros) y de las plantas.

En el nivel 2 encontramos ubicadas respuestas en las que de "una manera simple se reconocen las relaciones de cadenas tróficas; se reconoce la diversidad de seres vivos y producciones de los mismos, indicando su presencia en los ecosistemas, se admite que las relaciones ecológicas se dan a nivel de poblaciones" (García, 1999). En los resultados se encontró que los estudiantes relacionan el eco- sistema con la naturaleza y el medio que los rodea, donde incluyen animales y plantas. Algo muy importante que mencionan es la existencia de una relación entre estos; es decir, para ellos hay una interacción, pues los alumnos dibujaron algunos animales como las aves alimentando a sus crías, y otros alimentándose de animales ya muertos, como lo indica García (2003):

\begin{abstract}
También se identifica una mayor diversidad de relaciones ecológicas: relaciones entre la biocenosis y el biotopo, relaciones interespecíficas que no sea la relación depredador - presa (relaciones de cobijo entre seres vivos, relaciones tróficas menos evidente, relaciones de competencia; etc.), así como relaciones intraespecíficas (de carácter reproductivo, de crianza o que implican un cierto grado de asociación en relación con la localización o el cobijo. (p. 93).
\end{abstract}

Por otra parte, encontramos respuestas tales como relación entre seres vivos y muertos y cuando un animal se come a otro. Tal tipo de respuestas nos puede dar un breve acercamiento a lo que sería el nicho ecológico, así como sus relaciones con otras especies y por su forma de alimentación.

\section{Conclusiones}

Según la categorización de las ideas previas, el 26,1\% de las respuestas dadas por los estudiantes en la totalidad de preguntas hechas se ubicaron en el nivel 0 (no relación), mientras que el $71,17 \%$ se ubicó en el nivel 1 (ecosistema como medio aditivo) y el 7,08 \% restante se ubicó en el nivel 2 (ecosistema como organización simple); se logró evidenciar que ningún estudiante se ubicó en el nivel 3 , pues no proporcionaron respuestas que permitan dar cuenta de la complejidad de las relaciones ecológicas existentes.

En la primera pregunta, el $17,94 \%$ de los estudiantes se encuentran en el nivel 2 (ecosistema como organización simple), pero dicho porcentaje varía al momento en que se les cuestiona acerca de lo que contiene un ecosistema: vemos que no se ubican estudiantes en ese nivel. Por otra parte, el nivel 0 (no relación) aumenta en una proporción del 33,33\%: en él se ubican los estudiantes que mencionan objetos -por ejemplo, escritorios y cuadernos- como principales componentes de un ecosistema; cabe mencionar que tan solo el $33,33 \%$ dibuja rocas y esqueletos como parte de un ecosistema. En cuanto a los dibujos de cosas vivas, se encuentra que el $58 \%$ de los estudiantes plasma en sus dibujos a las personas, entendido el hombre como integrante de los ecosistemas. Para el ítem número 3 , donde se le pide al estudiante que dibuje un ecosistema, el nivel 0 (no relación) desaparece, y los 
educandos ya no realizan dibujos de objetos usados a diario; así, el nivel 2 (ecosistema como organización simple) varía en una proporción del $12,82 \%$, donde los estudiantes logran dar cuenta en sus dibujos de un mayor acercamiento a las relaciones ecológicas.

Esta investigación nos permite contemplar las ideas previas como la base de la construcción de nuevos conocimientos escolares, pues a partir de las experiencias propias de los estudiantes se adquieren ideas que son fundamentales al momento de aprender cosas nuevas, percibir el entorno e interpretarlo; frente al concepto ecosistema, se encuentran puntos clave en los alumnos ubicados en el nivel 2 (ecosistema como organización simple), de los cuales se puede profundizar para lograr una buena comprensión del concepto.

\section{Referencias}

Bello, S. (2004). Ideas previas y cambio conceptual. Departamento de Química Inorgánica y Nuclear, Facultad de Química, Unam. 04510. México, DF. Recuperado de: http://depa.fquim.unam.mx/sie/ Documentos/153-bel.pdf

Casillas C. y Ramos, M. (2012). Unidad Didáctica: El ecosistema. Recuperado de: http://es.slideshare. net/7Martes3/unidad-didctica-los-ecosistemas

Cubero, R. (1997). Cómo trabajar con las ideas de los alumnos. Cuarta edición, número 1. Sevilla: Diada serie práctica (Colección Investigación y enseñanza).

García, J. (1999). Una hipótesis de progresión sobre los modelos de desarrollo en educación ambiental. Investigación en la Escuela (37), 15-32. Universidad de Sevilla. Recuperado de: http://www.investigacionenlaescuela.es/articulos/37/R37_2.pdf

García, J. (2003). Investigando el ecosistema. Investigación en la Escuela (51), 83-100. Universidad de Sevilla. Recuperado de: http://www.investigacionenlaescuela.es/articulos/51/R51_7.pdf

Gil, P. (1993). II.2. Propuestas alternativas para la introducción de los conceptos científicos: de aprendizaje como cambio conceptual al aprendizaje como investigación. En: D. Gil y M. Guzmán (Eds.). Enseñanza de las Ciencias y la Matemática. Tendencias e Innovaciones. Organización de Estados Iberoamericanos. CEI para la Educación, la Ciencia y la Cultura. Ciencias. Editorial Popular, Universitat de València. Recuperado de: http://www.oei.es/ oeivirt/gil02b.htm
Gil, P. y Guzmán, M. (Eds.). (1993). Enseñanza de las Ciencias y la Matemática Tendencias e Innovaciones. Organización de Estados Iberoamericanos. CEI Para la Educación, la Ciencia y la Cultura. Ciencias. Editorial Popular, Universitat de València. Recuperado de: http://www.oei.es/oeivirt/ciencias.htm\#Indice.

Giordan, A. (1982). La enseñanza de las ciencias. Madrid, España: Siglo XXI. Recuperado de: https:// docs.google.com/document/d/1poF4K3A0eOPUvkAvqAMdWXOE-sV LUPe85kNUaX7WGg/edit?hl=es

Grilo, D., Mellado, P. y Ruiz, C. (2004). Evolución de las ideas alternativas de un grupo de alumnos portugueses de secundaria sobre fotosíntesis y respiración celular. Revista de Educación en Biología, 7(1), pp. 10-20.

Helm, H. (2000). Misconceptions about physical concepts among South African pupils studying physical science. South African Journal of Science 74, 285-290.

Ibarra, J y Gil, M. (2009) Uso del concepto de sucesión ecológica por alumnos de secundaria: la predicción de los cambios en los ecosistemas. Enseñanza de las Ciencias, 27(1), 19-32. Recuperado de: http://www.raco.cat/index.php/ensenanza/article/ viewFile/132204/332988

Leach, J., Driver, R., Scott, P. y Wood-Robinson, C. (1992). Progression in conceptual understanding of ecological concepts by pupils aged 5 to 16. Centre for Studies in Science and Mathematics Education. Leeds University.

López, R. (2002). Teoría de los Ecosistemas Ecológicos, 2da edición. España: Universidad de Barcelona.

Muñoz, C. (2005). Ideas previas en el proceso de aprendizaje de la historia. Caso: estudiantes de primer año de secundaria, Chile. Universidad de Concepción. Chile: Geoenseñanza, Vol. 10-2005-2.

Novak, J. (1983). Overview of the Seminar. En: Proceedings of the international Seminar: Misconceptions in science and mathematics Ithaca, N.Y. USA: Commell University Press.

Osborne, R. , Bell, F. y Gilbert, J. (1983). «Science Teaching and Children 's views of the world». en: European Journal Science Education. 5 (1), Egland.1-14

Perkins, D. y Blythe, T. (2005). Ante todo, la comprensión. Revista Magisterio Educación y Pedagogía 14, abril. Recuperado de: http://www.uca.edu.ar/ uca/common/grupo18/files/perkins_antetodo_la_ comprension.pdf 
Pozo, J. (1996). Las ideas del alumnado sobre la ciencia: de dónde vienen, a dónde van... y mientras tanto qué hacemos con ellas. Revista Alambique. Didáctica de las Ciencias Experimentales. Recuperado de: http://alambique.grao.com/revistas/alambique/007-las-ideas-del-alumnado-en-ciencias/ las-ideas-del-alumnado-sobre-la-ciencia-de-donde-vienen-a-donde-van--y-mientras-tanto-que-hacemos-con-ellas

Pozo, J., Asensio, M. y Carretero, M. (1989). Modelos de aprendizaje-enseñanza de la Historia. En: M. Carretero, J. Pozo y M. Asensio. La Enseñanza de las Ciencias Sociales (pp. 139-163). Madrid: Visor.

Rayas, J. (2002). Ideas previas sobre energía en niños y niñas de $5^{\circ}$ grado de educación primaria y sus opiniones acerca de las actividades de aprendizaje. Tesis de maestría, UPN. México. Recuperado de: https://docs.google.com/document/d/1poF4K3A0eOPUvkAvqAMdWXOE-sV-LUPe85kNUaX7WGg/edit
Ricoy, L. (2006). Contribución sobre los paradigmas de investigación. Revista do Centro de Educação, 31 (1), 11-22

Rincón, M. (2011). Concepciones de los estudiantes de educación básica sobre ecosistema. Una revisión documental. Recuperado de: http://revistas. pedagogica.edu.co/index.php/bio-grafia/article/ view/1756/1735

Rincón, M., Medellín, F. y Vargas, C. (2004). Concepciones sobre nociones ecológicas en niños de las escuelas rurales de Villeta (Cundinamarca). Informe CIUP. Bogotá. Recuperado de: revistas. pedagogica.edu.co/index.php/bio-grafia/article/.../1756/1735

Sánchez, F. y Pontes, A. (2009). La comprensión de conceptos de ecología y sus implicaciones para la educación ambiental. Revista Eureka sobre Enseñanza y Divulgación de las Ciencias. Recuperado de: http://reuredc.uca.es/index.php/tavira/article/ viewFile/45/44 